\title{
Editorial
}

\section{Resources for pressure injury practice during COVID-19 pandemic}

Haesler E

For referencing Haesler E. Resources for pressure injury practice during COVID-19 pandemic. Wound Practice and Research 2020; 28(2):48.

DOI https://doi.org/10.33235/wpr.28.2.48

I am pleased to present this pressure-injury themed issue of Wound Practice and Research, which follows publication of the third edition of the Prevention and Treatment of Pressure Ulcers/Injuries: Clinical Practice Guideline (known as the International Guideline) in November 2019. The papers presented in this issue focus on aspects of pressure injury prevention and treatment. Floyd et al. discuss the benefits and costs of conducting pressure injury prevalence auditing, while Boylan presents a focus on pressure injury prevention in children and neonates, an area that is under-addressed in the pressure injury research despite the vulnerability of little ones in the hospital environment.

During the planning for the journal issue we have experienced the unprecedented COVID-19 pandemic. In his paper, Gefen includes discussion of the intersection between COVID-19 and pressure injury aetiology, highlighting the way comorbidities can exacerbate the risk of pressure injury. Barakat-Johnson et al. provide a timely overview of pressure injury prevention for patients in prone position, which is recommended for managing COVID-19 patients with acute respiratory distress syndrome. Meanwhile, Coyer et al. remind us that health professionals themselves have been vulnerable to device related pressure injuries (DRPIs), and report on a practical tip sheet for clinical staff.

\footnotetext{
Emily Haesler

PhD, PGradDipAdvNurs (Gerontics), BN Adjunct Associate Professor, Western Australia Centre for Evidence Informed Healthcare Practice, Wound Healing and Management Unit, Curtin University, WA Honorary Senior Lecturer, The Australian National University, ANU Medical School, ACT Honorary Associate, Australian Centre for Evidence Based Aged Care, School of Nursing and Midwifery La Trobe University, Bundoora, VIC Email Emily.Haesler@anu.edu.au
}

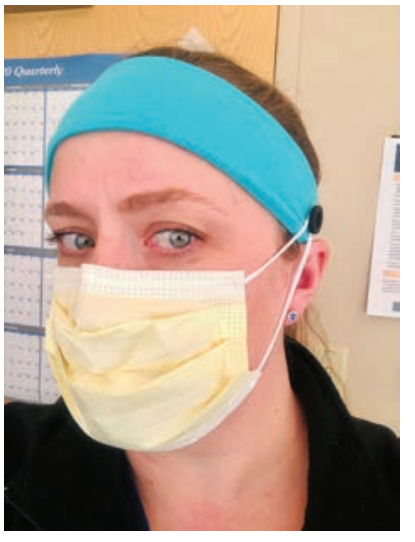

Photo courtesy Krista S. King (BSN, RN, CWOCN). Inpatient Wound Ostomy Team, University of Colorado Hospital, Aurora, Colorado, USA.

Used with permission.
During the COVID-19 pandemic, the international wound community has risen to many challenges and has rapidly developed recommendations, resources and supports. I draw your attention to COVID-19 resources accessible for free from the National Pressure Injury Advisory Panel website (www.npiap.org), and the European Pressure Ulcer Advisory Panel's Facebook Page - COVID-19 and pressure ulcer prevention forum - where wound clinicians are sharing their experience, resources and ideas. One such tip comes from WOCN nurses and their patients in Colarado US who have created a "button headband" to decrease the risk of pressure injuries associated with face masks and visors (see photo). However, when making adjustments to devices, the function of the equipment should not be compromised. The new 2019 International Guideline, that includes recommendations relevant to all patients, also provides guidance on preventing DRPIs. The guideline through links at www.internationalguideline.com.

The current pandemic provides us all with a stark reminder on the importance of infection control when treating wounds. The Wounds Australia Standards for Wound Prevention and Management (2016) includes fundamental infection control principles and procedures for wound practitioners that are relevant for use in conjunction with your organisation's COVID-19 guidelines.

On behalf of the Editor and Editorial Board, thanks to the contributors to this issue of WPR who have submitted manuscripts at a time of extreme upheaval for most of us, both professionally and personally. 